\title{
Preface of the Special Issue on Theoretical Aspects of Computer Science (2018)
}

\section{Rolf Niedermeier ${ }^{1} \cdot$ Brigitte Vallée $^{2}$}

Published online: 30 January 2020

(C) Springer Science+Business Media, LLC, part of Springer Nature 2020

This special issue contains eight articles which are based on extended abstracts that were presented at the 35th Symposium on Theoretical Aspects of Computer Science (STACS), which was held at the University of Caen from February 28th to March 3rd, 2018. These extended abstracts were chosen among the top papers of those that were selected for presentation at STACS 2018 in a highly competitive peer-review process (after which only 54 papers out of 186 submissions were accepted).

Compared with the original extended abstracts, the articles have been extended with a description of the context, full proofs and additional results. They underwent a further rigorous reviewing process, following the TOCS Journal standards, completely independent from the selection process of STACS 2018.

The topics of the chosen papers cover a rich set of areas within Theoretical Computer Science, that is, algorithmic game theory, automata theory, circuit complexity, efficient algorithms, logic and model checking, Markov chains, parameterized complexity, and proof complexity.

Kernelization (provably effective and efficient preprocessing) is a central theme in parameterized complexity. The article Computing Hitting Set Kernels by AC $C^{0}$-Circuits by Max Bannach and Till Tantau presents for the first time kernels for the d-HITTINF ET problem that can be computed in constant parallel time, thereby refuting a previous conjecture. To this end, the authors introduce a new, generalized notion of hypergraph sunflowers. Altogether, this gives an important contribution to parameterized circuit complexity.

Brigitte Vallée

brigitte.vallee@unicaen.fr

Rolf Niedermeier

rolf.niedermeier@tu-berlin.de

1 Fakultät IV, AKT, Technische Universität Berlin, Straße des 17. Juni 135, 10623 Berlin, Germany

2 GREYC, CNRS and Université de Caen Normandie, Espl. de la Paix, 14000 Caen, France 


\section{Rolf Niedermeier, Brigitte Vallée}

The article Lower Bound Techniques for QBF Expansion by Olaf Beyers-dorff and Joshua Blinkhorn presents new lower bound techniques for expansion-based QBF proof systems. These systems are motivated by recent developments in automated QBF solving. A particular appeal is the elegance of the lower bound proofs, simplifying previous proofs. All this is based on natural and general techniques for proving lower bounds.

The article On the Tree Conjecture for the Network Creation Game by Da-vide Bilô and Pascal Lenzner studies Nash equilibria and the price of anarchy in the classic sum model of Network Creation Games. These are motivated by applications in several scenarios including social and economic networks where decentralized processes have to be taken into account. The authors address open problems in the field and prove several new results, in particular introducing a novel technique for analyzing stable networks for high edge-price.

Approximating the stationary probability $\pi(v)$ of a state $v$ in a Markov chain is, in general, inefficient, and the best available methods have a complexity that depends inversely on $\pi(v)$, and are thus inefficient for small $\pi(v)$. The article On approximating the stationary distribution of time-reversible Markov chains by Marco Bressan, Enoch Peserico, and Luca Pretto exhibits, for time-reversible Markov chains, a simple randomized approximation algorithm whose complexity is independent of $\pi(v)$ and thus breaks the "small- $\pi(v)$ barrier".

Strategy Logic (SL) is a very expressive temporal logic, at the expense of a very high complexity. The paper Dependences in Strategy Logic by Patrick Gardy, Patricia Bouyer, and Nicolas Markey introduces timeline semantics and defines a large (and, in a sense, maximal) fragment of SL for which syntactic and semantic negations coincide under the timeline semantics. As an (important) side result, it shows that model checking this fragment under the timeline semantics is 2-EXPTIME-complete.

The paper Optimal dislocation with persistent errors in subquadratic time by Barbara Geissmann, Stefano Leucci, Chih-Hung Liu, and Paolo Penna studies the problem of sorting $N$ elements in the presence of persistent errors in comparisons (with probability $p$ ). A measure of quality is then the maximum dislocation, i.e., the maximum absolute difference between the position of an element in the returned sequence and the position of the same element in the perfectly sorted sequence. It is known that no algorithm can achieve maximum dislocation $o(\log N)$ with high probability. The best known algorithms for this problem have running time $O\left(N^{2}\right)$ and achieve, with high probability, an optimal maximum dislocation of $O(\log N)$ for constant error probability $p$. The paper presents the first subquadratic-time algorithm which runs in $O\left(N^{3} / 2\right)$ time and guarantees, with high probability, for any $p \leq 1 / 16$, a maximum dislocation of order $O(\log N)$.

The problem of finding a / the (length of a) longest increasing subsequence (LIS) of an integer sequence is a classical problem in computer science. The article SpaceEfficient Algorithms for Longest Increasing Subsequence by Masashi Kiyomi, Hirotaka Ono, Yota Otachi, Pascal Schweitzer, and Jun Tarui approaches the problem of finding an LIS in an array in a space-efficient fashion, while keeping the running time low as well. They show that the time complexity of their algorithms is optimal up to 
polylogarithmic factors in a restricted model. Altogether, these are the first spaceefficient algorithms for finding an LIS.

In the article Additive Number Theory via Automata Theory, Aayush Ra-jasekaran, Jeffrey Shallit, and Tim Smith, show how some problems in additive number theory can be attacked in a novel way, using techniques from the theory of finite automata. Recently, Cilleruelo, Luca, and Baxter proved, for all bases $b \geq 5$, that every natural number is the sum of at most three natural numbers whose base-b representation is a palindrome. However, the cases $b=2,3,4$ were left unresolved. The authors of the present paper prove that every natural number is the sum of at most four natural numbers whose base- 2 representation is a palindrome. They obtain similar results for bases 3 and 4, thus completely resolving the problem of palindromes as an additive basis.

To conclude, we express our deep gratitude to the authors for their contributions and to the referees for their helpful reports. We also want to thank the members of the Program Committee and the Steering Committee of the conference. Finally, we thank the members of the TOCS editorial office for the very smooth cooperation when preparing this special issue.

Rolf Niedermeier and Brigitte Vallée, Guest Editors, January 2020.

Publisher's Note Springer Nature remains neutral with regard to jurisdictional claims in published maps and institutional affiliations. 